\title{
A LOW-ANGLE X-RAY DIFFRACTION STUDY OF THE SWELLING BEHAVIOR OF PERIPHERAL NERVE MYELIN
}

\author{
C. R. WORTHINGTON AND A. E BLAUROCK
}

Department of Physics and Biophysics Research Division, University of Michigan, Ann Arbor, Mich. 48104 (U.S.A.)

(Recelved November 4th, 1968)

\section{SUMMARY}

The swelling behavior of peripheral nerve myelin has been studied by recording low-angle X-ray diffraction patterns of nerve after various chemical treatments. Peripheral myelin swells in distilled water, hypotonic Ringer's solution and sucrose solutions. The resulting low-angle pattern is called a swollen pattern (by comparison, the normal pattern is obtained from live nerve). The swelling of myelin is reversed by a reimmersion of the nerve in salt solutions and the resulting low-angle pattern is called a subnormal pattern. A relationship between these three kinds of patterns is given in terms of the width of the membrane pair. The forces between the adjacent membrane pairs in swollen myelin are likely to be long-range attractive Van der Waals' and long-range electrical repulsive forces. However, an equilibrium between the attractive and repulsive forces has not been observed.

\section{INTRODUCTION}

An important property of peripheral nerve myelin is its ability to swell in hypotonic Ringer's solutions*. Finean and Millington ${ }^{1}$ in a low-angle X-ray experiment first recorded a much larger than normal radial repeat period for frog sciatic nerve in $0.25 \times$ Ringer's solution. ROBERTSON ${ }^{2}$ in an electron-microscopic study has shown that a major part of this swelling takes place at the outer surfaces of the membrane pair (the two adhering plasma membranes). The occurrence of this swelling has been further verified by more recent $X$-ray diffraction studies ${ }^{\mathbf{3}, \mathbf{4}}$ but a detailed account of the $\mathrm{X}$-ray results has not been given.

The property of swelling is an important one in itself because a detailed study may provide information which could lead to an understanding of the forces between the adjacent membrane pairs in the normal and the swollen state. However, the property of swelling is important for another reason, namely, it suggests an obvious $X$-ray method ${ }^{5,6}$ which if correctly formulated and carried out, could lead to a structural interpretation of nerve myelin.

In this paper we describe $\mathrm{X}$-ray experiments relating to the swelling behavior of peripheral nerve myelin. The corresponding radial repeat distances are given and we note any new features in the low-angle $X$-ray patterns. No attempt has been made to give a structural analysis of the $\mathrm{X}$-ray data as our primary purpose at this time

* Ringer's solution $\mathrm{NaCl}, 9 \mathrm{~g} / 1, \mathrm{KCl}, 0.2 \mathrm{~g} / 1 ; \mathrm{CaCl}_{2}, 0.2 \mathrm{~g} / 1, \mathrm{NaHCO}_{3}, 0 . \mathrm{g} / 1$; the dilutions are given as a multiple of this. 
is to elucidate the factors which determine the swelling behavior of peripheral nerve myelin.

We identify (at least) three kinds of low-angle X-ray patterns. These are the normal pattern, the swollen pattern and the subnormal pattern. We have recently proposed an electron-density model for nerve myelin ${ }^{7}$ in the swollen state; one of the parameters of the model is the width or the thickness of the membrane pair. A possible relationship between these three patterns is given in terms of the membrane pair width.

\section{EXPERIMENTAL}

An account of the experimental methods and $\mathrm{X}$-ray equipment used was given in the previous paper ${ }^{8}$.

Specimens of peripheral nerve myelin were sciatic nerves from either frog (Rana pipiens) or rat. However, frog sciatic nerves were used in nearly all of the experiments.

The dissections, the insertion of the nerve specimens into capillary tubes and the $\mathrm{X}$-ray experiments were carried out at room temperature $\left(20^{\circ}\right)$. The immersion of the specimens in various solutions was carried out, either at $4^{\circ}$, or at room temperature $\left(20^{\circ}\right)$.

The error in measuring the radial repeat distance is estimated to be $I \%$, although this can be reduced to $0.5 \%$ by using a larger specimen-to-film distance ${ }^{8}$. The radial repeat distances quoted in this paper are accurate to within at least $\mathrm{I} \%$.

\section{Normal pattern of peripheral nerve myelin}

Peripheral nerve myelin in the normal state refers to a freshly dissected nerve specimen in I $\times$ Ringer's solution. The radial repeat distance, $d$, or simply the perıod, $d$, is measured directly from the recorded low-angle X-ray pattern; $d=\mathrm{I} 7 \mathrm{I} \AA$ for frog sciatic nerve, $d=I_{7} 6 \AA$ for rat sciatic nerve. Photographs of these patterns have been shown in the previous paper ${ }^{8}$.

\section{Peripheral nerve myelin in distilled water}

The immersion time for peripheral nerve myelin in distilled water was from $\mathrm{I}_{2}-\mathrm{I}_{4} \mathrm{~h}$. The low-angle $\mathrm{X}$-ray patterns showed that the period, $d$, had increased by at least $80 \AA$. Three frog sciatic nerve specimens (Specimens I, 2 and 3 ) were immersed at $4^{\circ}$ for various times and gave a small range of periods, $d=25^{2-264} \AA$. Another frog sciatic nerve specimen (Specimen 4 ) was immersed in distilled water for $30 \mathrm{~h}$ at $20^{\circ}$ and showed an even larger period, $d=342 \AA$. A rat sciatic nerve specimen was immersed in distilled water for about $40 \mathrm{~h}$ at $20^{\circ}$ and showed a period, $d=274 \AA$. The radial repeat distances of frog sciatic nerve corresponding to the different immersion times in distilled water and the temperatures are given in Table $\mathrm{I}$. The X-ray pattern obtained from Specimen I in Table I which has $d=252 \AA$ is shown in Fig. I. The diffraction orders $h=\mathrm{I}, 3,4,6,7$ are readily seen, while orders $h=5,8$ which cannot be seen in Fig. I are, however, visible on the original X-ray film. The exposure time was $4 \mathrm{~h}$. This kind of peripheral nerve myelin pattern is called a swollen pattern. The nature of the swollen pattern differs from the normal pattern, not only because of the increased period, but because the widths of the diffraction orders show a marked broadening with increase in diffraction order $(h)$. We note that the swollen 


\section{TABLE I}

IMMERSION TIMES, TEMPERATURES AND RADIAL REPEAT DISTANCES FOR FROG SCIATIC NERVE IN DISTILLED WATER

\begin{tabular}{llcl}
\hline Specimen & Immersion time $(h)$ & Temp. & $d(A)$ \\
\hline I & 24 & $4^{\circ}$ & 252 \\
2 & I 4 & $4^{\circ}$ & 264 \\
3 & I 2 & $4^{\circ}$ & 254 \\
4 & 30 & $20^{\circ}$ & $34^{\circ}$ \\
\hline
\end{tabular}

myelin period in distilled water, unlike that of normal myelin, is not a constant reproducible quantity. The swollen myelin period shows a small variation in period; two closely similar experiments will generally show periods which are not exactly the same.

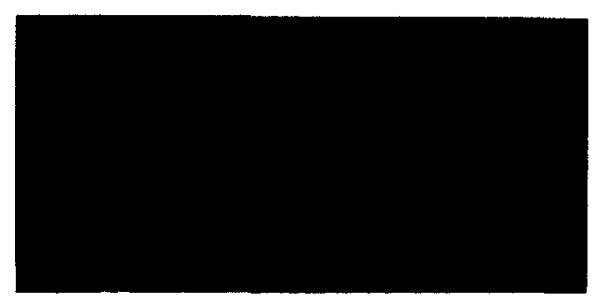

Fig. I. Low-angle X-ray diffractıon pattern of frog scıatıc nerve in distılled water (24 hours at $4^{\circ}$ ). The pattern was taken at $20^{\circ}$ using $30 \mathrm{kV}$ copper $\mathrm{K} \alpha$ nickel-filtered radiation, a specimen-to-film distance of $13.2 \mathrm{~cm}$ and an exposure time of $4 \mathrm{~h}$. This is a swollen pattern with a radial repeat distance of $d=252 \AA$ Diffraction orders $h=\mathbf{I}, 3,4,6,7$ are visible, however, the weaker orders, $h=5,8$, which cannot be seen in the reproduction, are visible on the original X-ray film. Order $h=I$ is comparatively sharp, but the higher orders are less sharp. The line width increases with increasing diffraction order.

\section{Frog sciatic nerve in hypotonic Ringer's solution}

Frog sciatic nerve was immersed in various hypotonic Ringer's solutions for a period of about I day at $4^{\circ}$ and the X-ray pattern recorded at $20^{\circ}$. The normal pattern for frog sciatic nerve is obtained with $\mathrm{I} \times$ Ringer's solution and $d=\mathrm{I} 7 \mathrm{I} \AA$. The case of zero concentration has been described in the above section, a swollen pattern is recorded (see Table I) with no trace of the normal pattern. When the concentration of Ringer's solution is increased from zero to $0.3 \times$ only the swollen pattern is obtained. The radial repeat distances for frog sciatic nerve in $0.1-0.3 \times$ Ringer's solution is given in Table II. The swollen periods range from $d=258$ to $280 \AA$ for the concentration range of $0 . \mathrm{I} \rightarrow 0.3 \times$ Ringer's solution, and this compares favorably with an earlier observation ${ }^{1}$ of $d=270 \AA$ for frog sciatic nerve in $0.25 \times$ Ringer's solution. The case of $0.5 \times$ Ringer's solution was studied. In one experiment the normal pattern was obtained, but in another experiment, a swollen period, $d=240 \AA$, was superimposed on the normal pattern. The concentration $0.5 \times$ Ringer's solution seems to be border line for swelling.

The swollen patterns obtained with hypotonic Ringer's solutions show an increase in line broadening with an increase in diffraction order similar to the swollen pattern recorded using distilled water (see Fig. I). 
TABLE II

RADIAL REPEAT DISTANCES FOR FROG SCIATIC NERVE IN HYPOTONIC RINGER'S SOLUTIONS

\begin{tabular}{lll}
\hline Spectmen & Concn. & $d(A)$ \\
\hline & & \\
5 & $0.1 \times$ & 258 \\
6 & $0.2 \times$ & 280 \\
7 & $0.3 \times$ & 262 \\
\hline
\end{tabular}

We note that no X-ray pattern intermediate between the normal and swollen pattern was obtained. When the tonicity of the Ringer's solution is reduced below $0.5 \times$, there is an abrupt jump in the radial repeat distance of at least $80 \AA$. The swollen period does not correlate with the concentration of the Ringer's solution, for example the swollen period (Table II) is about the same for both 0.I and $0.3 \times$ Ringer's solution.

\section{Reversal of swelling in distrlled water}

The procedure adopted was to immerse frog sciatic nerve in distilled water at $4^{\circ}$ until swelling occured. Then the swollen nerve specimens were immersed in various salt solutions at $4^{\circ}$ for about $\mathrm{I} 2 \mathrm{~h}$ before recording the low-angle pattern (at $20^{\circ}$ ). Various salt solutions which reversed the swelling in distilled water are listed below. The pattern obtained was different from the normal pattern in that the radial repeat period was smaller by about $5 \AA$ and the intensity variation differed significantly. This pattern is called a subnormal pattern. A typical subnormal pattern is shown in Fig. 2. The nerve specimen was immersed in distilled water for $\mathrm{I} 2 \mathrm{~h}$ (similar to Specimen 3 in Table I) and later put in $2 \mathrm{mM} \mathrm{CaCl}_{2}$ solution at $4^{\circ}$ for $\mathrm{I} 2 \mathrm{~h}$. An exposure time of $I \mathrm{~h}$ was used to record the pattern. The period of the nerve specimen was

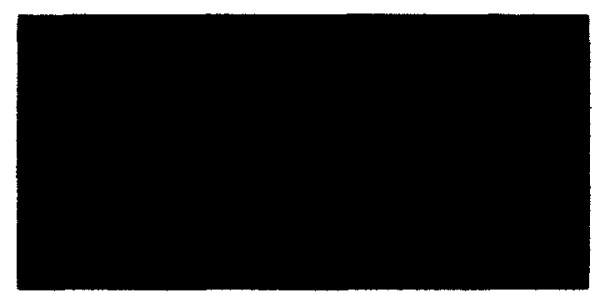

Fig. 2. Low-angle X-ray diffraction pattern of frog sciatic nerve in $2 \mathrm{mM} \mathrm{CaCl}_{2}$ solution (I 2 h in distilled water at $4^{\circ}$ followed by $12 \mathrm{~h}$ in $2 \mathrm{mM} \mathrm{CaCl}_{2}$ solution at $4^{\circ}$ ). The pattern was taken at $20^{\circ}$ using $30 \mathrm{kV}$ copper $K \alpha$ nickel-filtered radiation, a specimen-to-film distance of $13.2 \mathrm{~cm}$, and an exposure time of $\mathrm{I} h$. This is a subnormal pattern with a radial repeat distance of $d=\mathrm{I} 65 \AA$. Diffraction orders $h=2,3,4,5$ are visible. Note the sharpness of the diffraction lines which are comparable to the normal pattern.

$d=165 \AA$. The intensity variation differs from the normal pattern in that $I(3) \approx I(4)$ where $I(h)$ is the intensity of diffraction order $h$. The diffraction lines in the subnormal pattern have a narrow line width and in this respect, are almost as sharp as the diffraction lines in the normal pattern ${ }^{8}$. If the nerve specimen is allowed to stand in the particular salt solution, either at $4^{\circ}$, or $20^{\circ}$, for a longer time (that is, longer than I2 h) then the repeat distance gradually increases to that of the normal pattern $d$ $=\Upsilon_{7} \AA$, that is, the subnormal pattern slowly transforms to the normal pattern. 
Various salt solutions which reversed the swelling in distilled water are: $\mathrm{I} \times$ Ringer's solution, I $\times$ Ringer's solution $\left(\mathrm{CaCl}_{2}\right.$ free), o.II $\mathbf{M ~ N a C l}$, o.II $\mathbf{M ~ K C l}$, o.I2 and $0.25 \mathrm{M} \mathrm{LiCl}, 0.12$ and $0.25 \mathrm{M} \mathrm{CsCl}$, I $\mathrm{mM}$ and $2 \mathrm{mM} \mathrm{CaCl}_{2}$, and I $\mathrm{M}$ Sorenson's phosphate buffer solution ( $\mathrm{pH} 7$ ). Thus, swelling was reversed by $\operatorname{o.II} \mathrm{M} \mathrm{NaCl}$ and o.II $\mathrm{M} \mathrm{KCl}$ solutions, but we also record that swelling was not reversed by ro $\mathrm{mM}$ $\mathrm{NaCl}$ or by Io $\mathrm{mM} \mathrm{KCl}$. The minimum concentration for the swelling to be reversed for $\mathrm{NaCl}$ and $\mathrm{KCl}$ is somewhere between Io and I Io $\mathrm{mM}$ but no real attempt was made to find the exact minimum concentration. It is also apparent from the above list that $\mathrm{CaCl}_{2}$ solution is most effective in bringing about the reversal of swelling in that only I mM concentration is needed. This parallels an earlier observation ${ }^{9}$ that $\mathrm{CaCl}_{2}$ is most effective in reversing the swelling in lipid emulsions.

We note that the low-angle $\mathrm{X}$-ray pattern obtained after the reversal of swelling is the subnormal pattern, and there is no trace of either the swollen pattern or the normal pattern. The subnormal pattern shows a small variation in the period $d$ $=\mathrm{I} 64-\mathrm{I} 68 \AA$; the experimental value of $d$ obtained from the pattern is a precise one, but its actual magnitude can change slightly depending on specimen preparation. If the nerve specimen is allowed to stand in the salt solution for a longer period of time then the subnormal pattern changes over to the normal pattern.

\section{Frog sciatic nerve in hypertonic Ringer's solution}

The low-angle patterns obtained when frog sciatic nerve was immersed in 2 and $4 \times$ hypertonic Ringer's solution showed a period $d=I 67 \AA$. The X-ray reflections $h=\mathrm{I}-5$ occurred as sharp lines similar to the normal pattern but differed from the normal pattern, not only by having a smaller period, but in that $I(3) \approx I$ (4). Hence these two low-angle patterns are identical to the subnormal pattern described in the above section. However, a weak diffuse reflection at $64 \AA$ which was not a diffraction order of $d={ }_{1} 67 \AA$ could also be seen in each of the two subnormal patterns. When the tonicity was increased to $7.7 \times$, the low-angle pattern obtained was not a subnormal pattern, for a period $d=I 84 \AA$ was recorded together with a reflection at $66 \AA$ which had strong intensity (much stronger than the 64 - $\AA$ reflection in the case of the 2 and $4 \times$ tonicity). The period $d=184 \AA$ for $7.7 \times$ agrees fairly well with an earlier observation ${ }^{1}$ of $d=$ rgo $\AA$ and a 68 - $\AA$ strong reflection for frog sciatic nerve in Io $\times$ Ringer's solution.

\section{Frog sciatic nerve in sucrose solutions}

It is possible that the swelling of peripheral nerve myelin that takes place in distilled water might be thought to arise as the result of reduced osmotic pressure. If this is the case, then if the distilled water is replaced by either an isotonic or hypertonic solution, one would expect the swelling to be reversed. ROBERTSON ${ }^{10}$, in an electron-microscopic study has used 4 and 6 times isotonic sucrose solutions to replace the distilled water in swollen frog sciatic nerve, but found no reversal of swelling, although in the case of ro times isotonic sucrose, he considered that this solution gave a slight reversal of swelling. Hence, it follows from this electronmicroscopic study that osmotic pressure is not an important factor in the swelling of peripheral nerve myelin. However, it is desirable that this conclusion and the electron-microscopic observations be verified by $\mathrm{X}$-ray diffraction studies.

Frog sciatic nerve specimens were first swollen in distilled water at either $4^{\circ}$ 
or $20^{\circ}$ and then immersed in $0.24,0.82$ and $\mathrm{I} .80 \mathrm{M}$ sucrose solutions at either $4^{\circ}$ or $20^{\circ}$. The radial repeat distances obtained and the specimen treatment details are given in Table III. The low-angle pattern obtained in the first experiment, ı.e., using the first specimen treatment given in Table III, is shown in Fig. 3. There are thirteen diffraction orders $(h=\mathrm{I}-\mathrm{I} 3)$ of $d=388 \AA$ visible on the X-ray film. The exposure time was I 2 h. In Fig. 3 orders $h=2,4,5,6,7$ are readily seen, but the orders $h=9$,

TABLE III

RADIAL REPEAT DISTANCES FOR FROG SCIATIC NERVE IN SUCROSE SOLUTIONS

\begin{tabular}{lll}
\hline Treatment & & $d(A)$ \\
\cline { 1 - 2 } Distilled water & Sucrose & \\
\hline & & \\
$9 \mathrm{~h}$ at $4^{\circ}$ & I $7.5 \mathrm{~h}$ in o $24 \mathrm{M}$ at $4^{\circ}$ & 388 \\
I I h at $4^{\circ}$ & I $45 \mathrm{~h}$ in $0.24 \mathrm{M}$ at $4^{\circ}$ & 350 \\
I $2 \mathrm{~h}$ at $4^{\circ}$ & I $2 \mathrm{~h}$ in o.82 M at $4^{\circ}$ & 359 \\
22 h at $20^{\circ}$ & $50 \mathrm{~h}$ in I $80 \mathrm{M}$ at $20^{\circ}$ & 426 \\
\hline
\end{tabular}

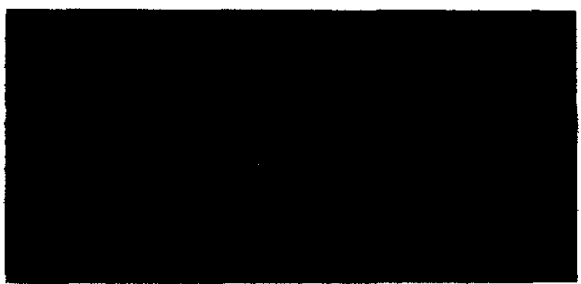

Fig. 3. Low-angle X-ray diffraction pattern of frog sciatic nerve in $0.24 \mathrm{M}$ sucrose solution ( $9 \mathrm{~h}$ in distilled water at $4^{\circ}$ followed by $175 \mathrm{~h}$ in $0.24 \mathrm{M}$ sucrose solution at $4^{\circ}$ ) The pattern was taken at $20^{\circ}$ using $30 \mathrm{kV}$ copper $\mathrm{K} \alpha$ nickel-filtered radiation, a specimen-to-film distance of $13.2 \mathrm{~cm}$ and an exposure time of $12 \mathrm{~h}$ This is a swollen pattern with a radial repeat distance of $d=388 \AA$ Diffraction orders $h=2,4,5,6,7$ are visible, orders $h=9$, Io, I I can also be seen but are not well resolved. Order $h=2$ is comparatively sharp, but the higher orders show marked line broadening, the line width shows a considerable increase with increasing diffraction order

Io, II tend to overlap or merge together. The low-angle patterns of myelin in sucrose solutions (after swelling in distilled water) are similar to the swollen patterns of myelin in distilled water. However, the radial repeat periods are larger than the case of distilled water alone, and the diffraction orders show a larger amount of line broadening with increase in period. This is shown in Figs. I and 3. We compare the same region, $d \approx 40 \AA$, in each case in Fig. I orders $h=6,7$ are well-defined, whereas in Fig. 3 the diffraction orders $h=9$, IO, II tend to merge together.

After immersing pre-swollen frog sciatic nerve in sucrose solutions, increased swelling ( $d=35^{0-426} \AA$ ) is recorded. An experiment on freshly dissected frog sciatic nerve, that is without any previous treatment with distilled water, was also carried out. A nerve specimen was put directly into $0.24 \mathrm{M}$ sucrose solution at $4^{\circ}$ for I3 $\mathrm{h}$ and the low-angle pattern recorded. A swollen pattern was obtained with $d=293 \AA$. Hence frog sciatic nerve swells in $0.24 \mathrm{M}$ sucrose solution.

If osmotic pressure was the cause of swelling in distilled water, then the isotonic (about $0.24 \mathrm{M}$ ) and the hypertonic sucrose solutions used in our experiments should have significantly reduced the swelling. However, the low-angle $\mathrm{X}$-ray patterns 
obtained in this study show the reverse. Hence our X-ray diffraction studies on frog sciatic nerve in sucrose solutions support the earlier electron-microscopic observations ${ }^{10}$ and confirm the conclusion that osmotic pressure plays little or no part in the swelling of peripheral nerve myelin.

\section{DISCUSSION}

In the course of this study at least three kinds of peripheral nerve myelin lowangle X-ray diffraction patterns have been identified: the normal pattern from live nerve or nerve in I $\times$ Ringer's solution ${ }^{8}$, the swollen pattern shown in Figs. I and 3 and the subnormal pattern shown in Fig. 2. The low-angle patterns obtained from frog sciatic nerve in hypertonic Ringer's solutions do not fit ideally into this scheme; we record either a subnormal pattern but with a weak diffuse reflection at $64 \AA$, or if the tonicity is high enough, a slightly swollen pattern together with a strong reflection at $66 \AA$.

A relationship between the three kinds of low-angle patterns (normal, swollen and subnormal) can be given. In a recent letter ${ }^{7}$ we proposed an electron-density model for peripheral nerve myelin in the swollen state. The model contains the familiar triple-layered unit for the plasma membrane; two such units together represent the membrane pair, and the pertinent model parameter is the width $(w)$ of the membrane pair. The model has a fluid layer of width $(d-w)$ between the outer surfaces of the membrane pair. The use of electron density strip models to account for the low-angle X-ray diffraction data from membrane-type structures has been described ${ }^{11}$. Model parameters for peripheral nerve myelin in the swollen and normal state have been given?. Details of the structure analysis, including the assignment of model parameters to peripheral nerve myelin in the subnormal state, is treated in a later publication ${ }^{12}$. In the swollen state, e.g., frog sciatic nerve in distilled water or in $0.24 \mathrm{M}$ sucrose solution, the width of the membrane pair is $w=\mathrm{I}_{45}$ and $\mathrm{I}_{42} \AA$, respectively. In the normal state, i.e., frog sciatic nerve in $\mathrm{I} \times$ Ringer's solution, $\mathrm{a}$ larger width is needed, $w=\mathrm{I}_{55} \AA$, and this leaves a fluid layer of I6 $\AA$. Therefore, when frog sciatic nerve pre-swollen in distilled water is immersed in salt solutions, the fluid layer in the swollen state, which is at least roo $\AA$ wide, is reduced to a smaller width approaching that in the normal state. The width of the membrane pair in the subnormal state is $w \approx I 45 \AA$, i.e., almost the same as in distilled water. The subnormal period of $d={ }_{1} 65 \AA$ therefore contains a fluid layer of about $20 \AA$. The slow transformation of the subnormal pattern to the normal pattern, then, involves an increase in $w$ and a slight decrease in the width of the fluid layer between the adjacent membrane pairs. Therefore, the actual value of the subnormal period $(d \approx I 64-I 68 \AA)$ for frog sciatic nerve depends on how far the slow transformation has gone.

The experiments with sucrose solutions essentially rule out the possibility that swelling is the result of osmotic pressure. Salt solutions clearly play an important role in determining the swelling behavior. Swelling results when salt solutions which are normally present in nerve myelin are diluted below a minimum concentration, and this swelling can be reversed by adding a variety of salt solutions.

It might be supposed that the ordering of the cylindrical layering in nerve is maintained by a balance of forces; Van der Waals' attractive forces and electrical repulsive forces. The presence of salt solutions in the fluid layer introduces a screening 
effect, and this reduces the electrical repulsion. Hence, one might expect a balance of forces to occur with only a small separation between the membrane pairs (our electrondensity model has a I6-A fluid layer). If the salt solution is diluted, then the electrical repulsion increases, hence the width of flurd layer increases until a new balance of forces is attained. The electrical forces are now of long range and therefore much weaker. The concept of long-range forces in brological systems is not new. Muscle is one particular system in which long-range forces are thought to maintain the filament lattice ${ }^{13}$ and there is experimental evidence ${ }^{14}$ in support of this concept. This description of forces most likely applies to myelin but there is a difference. The swelling of myelin has been found to take place abruptly, suggesting that a balance of forces may not occur. For example, frog sciatic nerve does not swell in hypotonic Ringer's solution until the tonicity is reduced below $0.5 \times$, and then it swells abruptly by at least $80 \AA$. Further reduction in tonicity changes the swelling only slightly if at all, and the swollen period does not correlate with the tonicity of the Ringer's solution.

The swollen pattern shows a marked line broadening with increase in diffraction order. The ordering of the cylindrical layers in the swollen state shows a certain amount of disorder, whereas normal myelin has very little disorder ${ }^{8}$. The ordering of the layers also seems to be dependent on the size of the period, i.e., a larger period shows more disorder. This would be in agreement with the concept of weaker attractive and repulsive forces with larger periods. However, our failure to observe an equilibrium, or to correlate the period with some parameter such as the ionic concentration, suggests that the magnitude of swelling may be dependent on the biological tissue supporting the myelin sheaf. The nerve axon tends to prevent myelin swelling inwards, whereas the connective tissues tend to prevent swelling outwards. In the absence of a balance of forces between the membrane pairs, the external environment then restricts swelling, and this leads to a new equilibrium, but an equilibrium which is less precise as the swollen period increases.

As noted in the INTRODUCTION, the property of swelling of myelin suggests an obvious X-ray method ${ }^{5,6}$ which was first used with hemoglobin crystals. In order to apply this method a basic assumption is needed. This states that during swelling the molecular structure of the membrane pair remains unchanged, and swollen myelin differs from normal myelin only in the width of the fluid layer between the adjacent membrane pairs. Our analysis ${ }^{72}$ determines that in the swollen state, the width of the membrane pair decreases IO-I5 $\AA$, and this naturally leads to some molecular changes within the membrane parr. This then contradicts the basic assumption, and, therefore, this X-ray method cannot be directly applied to nerve myelin, i.e., the sampling of a unique transform of nerve myelin is not obtained This does not mean, however, that $\mathrm{X}$-ray studies on swollen nerve are not worthwhile simply because an obvious method cannot be used. In fact, a systematic study of the X-ray patterns of peripheral nerve myelin in various stages of swelling is still likely to prove helpful in attaining a structural interpretation of live nerve.

\section{ACKNOWLEDGEMENT}

This work was supported by United States Public Health Service Grant GM09796. 


\section{REFERENCES}

I J. B. Finean and P F. Millington, J. Biophys. Biochem. Cytol., 3 (1957) 89.

2 J. D. Robertson, J. Buophys. Brochem. Cytol., 4 (I958) 349.

3 J. B. Finean and R E. Burge, $J$. Mol Biol, 7 (i963) 672.

4 M. F Moody, Sclence, I 42 (I963) II 73.

5 J. Boyes-Watson and M. F. Perutz, Nature, 15 I (1943) 714.

6 M. F. Perutz, Proc Roy Soc London, Ser. A, 225 (1954) 264.

7 C. R. Worthington and A. E. Blaurock, Nature, 2 I 8 (1968) 87.

8 A. E. Blaurock and C. R Worthington, Blochım. Brophys Acta, I73 (1969) 4 I9.

9 K. J. Palmer and F. O Schmitt, I Cellular Comp. Physiol, 17 (I94I) 385.

io J. D. Robertson in, M Locke, Cellular Membranes in Development, Academic Press, New York, I963.

I I C R. Worthington, Brophys J, 9 (I969) 222.

12 C. R. Worthington and A E. Blaurock, Biophys. $J$., in the press

I3 C. R. Worthington, $J$ Mol. Biol., 3 (I96r) 6r8.

14 G. F Elliot, $J$ Gen. Physiol, 50 (1967) I7т.

Brochim. Brophys. Acta, I 73 (1969) 427-435 